\title{
A Multi Agent Framework for Trading on the Energy of Internet
}

\author{
Qiang Luo, Chen Qi and Jinglin Guo \\ Beijing China Power Information Technology Co., Ltd., State Grid Electric Power Research Institute, Haidian District, Beijing \\ 100192, China
}

\begin{abstract}
We research and develop cloud based multi-functional smart agent technology with deep learning capability, provide technical solution for renewable energy in the hybrid Grids when microgrid integrated into State Grid, and accelerate evolution of the Energy of Internet in China.
\end{abstract}

\section{Keywords-micro grid, multi agent, Energy of Internet}

\section{INTRODUCTION}

In China, the micro-grid has been gradually moving from state-led investment, large-scale demonstration projects to policy-driven small investment, small-scale, more rational market-oriented business model. In the early stage, as the micro-grid is dominated by large state-owned national or demonstration project, the payback of investment is ineffective and needs to be made up by the environmental value analysis. However, the 21st century is an era that human use new energy in a large-scale. Countries are catching up with each other in the use of new energy sources and expect the right to dominate the use of new energy. In this context, Chinese micro-grid development considers more on the long-term interests.

At present, China only has made provisions for distributed energy generation, clearly limiting its capacity and direction of power flow. The main reason is the unreliability and poor stability of current (micro-grid) distributed energy generation, and that the impact for large grid safety has not really resolved. Another reason is the lag of research on standards. To make micro-grid large-scale apply in a more coordinated manner, it is urgent to develop standardization. The standardization of micro-grid involves the component products of micro-grid, or even the establishment of the internal structure of a typical micro-grid. From the law of grid development, the micro-grid will certainly walk into the road of homogenization as well.

China National Energy Board issued "Guidance on the establishment of renewable energy development and utilization objective guidance system " ( March 3, 2016 ) and decided the portion indicators of Non-hydro renewable energy power consumption out of the total electricity consumption in each provinces in 2020. The Guidance suggested that the energy development plan of every province should put the development and utilization of renewable energy as an important indicator, determine the portion indicator of renewable energy out of the total energy consumption and the non-hydro renewable energy power out of the social electricity consumption and make Renewable energy development and utilization plan accordingly.

\section{BASIC IDEA}

Micro grids offer an attractive path to rapid deployment of clean energy solutions. For example, they can be deployed rapidly and with relatively low investment requirements, thus reducing investment risks. However, effective management of micro grids can be challenging - with relatively small numbers of assets, they may lack the diversity and flexibility that regional and national grids can access in order to exercise control over grid operating state and stability. This problem is exaggerated when the micro grid contains a high proportion of clean but relatively inflexible assets, such as solar and wind generation. Thus effective use of intelligent control algorithms and "non traditional" control vectors (e.g. demand side response) is critical to widespread deployment of micro grids. This paper will develop such algorithms, focusing on multi-level algorithms that exploit a wide range of control vectors in order to optimise micro grid performance and stability. In particular, it will develop mechanisms for deploying local, highly responsive control algorithms that can tune a micro grid's performance through rapid control of distributed energy resources, combined with centralised "big data" machine learning loops that use global awareness to constantly tune and refine the local algorithms. These algorithms will address micro grid issues such as frequency regulation, management of voltages and reactive power, shifting and smoothing daily and seasonal demand peaks.

The paper research and develop multi-functional smart agent technology with deep learning capability and to advance the flexibility and adaptability in renewable energy enabled Energy of Internet. To provide optimized technical solutions for energy generation, transfer, trading and consumption.

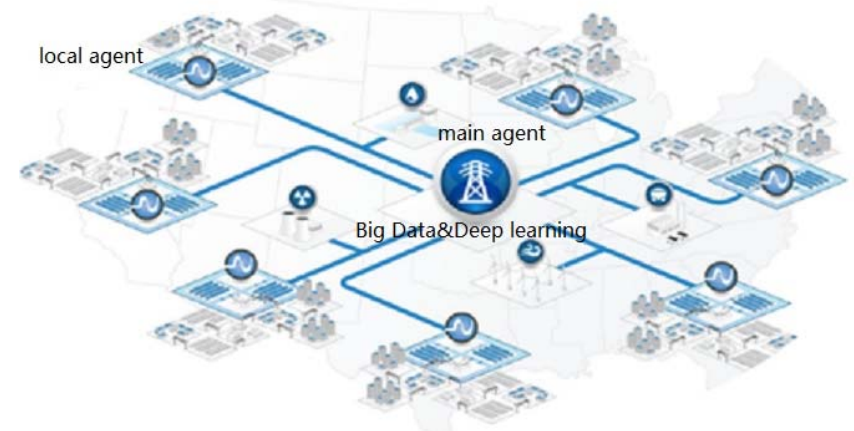

FIGURE I. MULTI AGENT ARCHITECTURE OF MICRO GRID

There are two basic ways of operation: Collaborative and Competitive. The use of Multi agent technology in controlling a 
MicroGrid solves a number of specific operational problems ${ }^{[1]}$. First of all, small Distributed Generation units have different owners, and several decisions should be taken locally, so centralized control is difficult. Furthermore, Micro grids operating in a market require that the actions of the controller of each unit participating in the market should have a certain degree of intelligence. Finally, the local DG units next to selling power to the network have also other tasks: producing heat for local installations, keeping the voltage locally at a certain level or providing a backup system for local critical loads in case of main system failure. These tasks suggest the importance of distributed control and autonomous operation.

\section{IMPLANTATION OF DATA ANALYSIS}

\section{A. Architecture Design}

Talia proposed that we can offer cloud computing data mining services from four levels ${ }^{[2]}$ : the bottom layer forms the basic steps of data mining algorithm; The second layer is a separate data mining service, such as classification, clustering, etc.; The third layer is distributed data mining models, such as parallel sorting, machine learning of aggregate type, etc.; the fourth layer is data mining applications based on the previous three layers. On the basis of such design, a data mining open service framework based on cloud computing is designed, and a series of data mining service systems have been developed. At the same time, users can define their own workflow through the graphical interface, and then perform it on the platform.

In this paper, the architecture above forms to the calculating layer. In addition, the whole work also includes the tools of query layer and storage layer, which aims to provide corresponding services for different users.

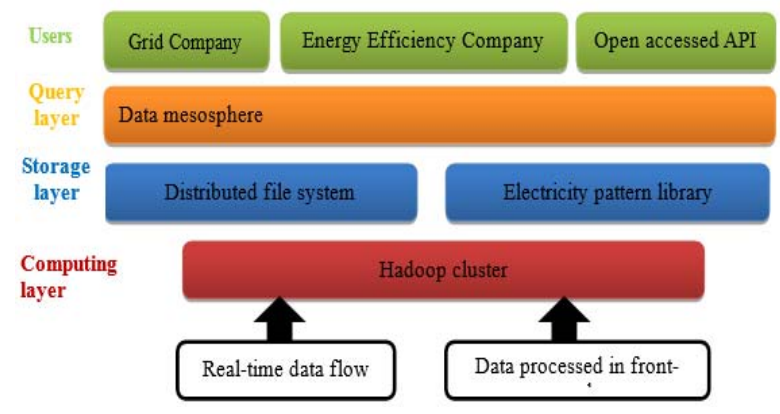

FIGURE II. DATA MINING ARCHITECTURE OF MICRO GRID

\section{B. Speed up the Data Analysis and Calculation}

Traditional data calculation generally is with a three layers structure, including compute nodes, data nodes and optical fiber network. The advantage of this approach is that it is very popular and easy to work. Users can build Hadoop cluster based on this architecture quickly. But in fact, this kind of structure restricts the performance of Hadoop greatly. In this work, PCIe2.0 communications and FPGA acceleration were adopted. Practice shows that our method calculating speed is about 10 times faster than traditional three-layer structure in the same configuration, data computing node, and under the condition of equal amount of data.

\section{Computation Acceleration Based on FPGA}

Both GPU and FPGA are common algorithm acceleration technologies ${ }^{[3,4]}$. In terms of current technology development, the comparisons between GPU and FPGA are as follows:

- GPU: strong parallel computing ability. And the use of high-level language can make developers focus on the research and implementation of algorithm. The disadvantage is high energy consumption and not fit for large scale use.

- FPGA: strong parallel computing ability. The technology implementation is difficult and each program statement presents actual hardware module. The advantage is low energy consumption and conducive to large scale use.

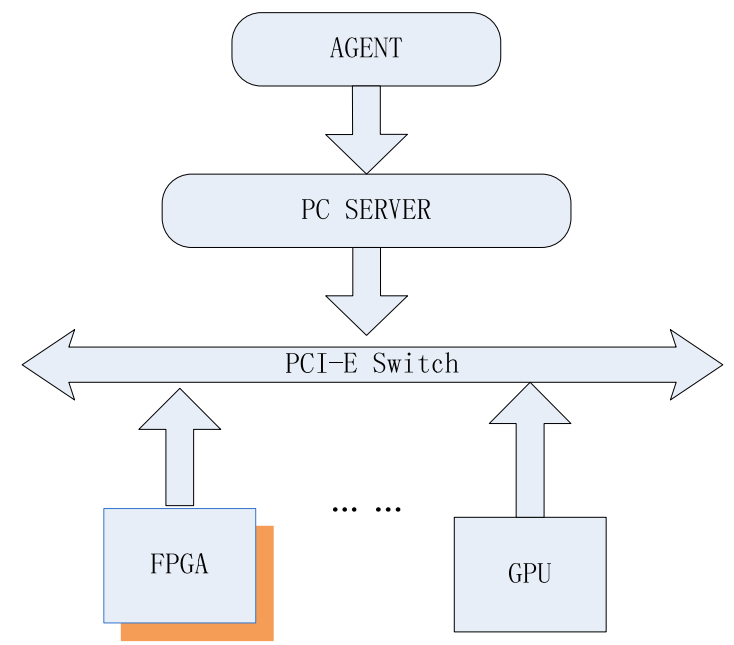

FIGURE III. REALIZATION OF DATA ACCELERATION ADOPTING FPGA AND GPU

Hence we can see that GPU is suitable for processing complex algorithms. FPGA can also implement complex algorithms since DSP and ARM module are added to the high-end devices of FPGA. Although the power meter pricing data in this work is large, the algorithm is relatively simple, so we use FPGA acceleration. Because pricing formula is not the same for users, we need to consider both task allocation and parallel computing method. Furthermore, there exits 20-80 rule in power meter pricing, that is, $80 \%$ calculations use $20 \%$ pricing formulas. A Convolutional Neural Network (CNN) is used in trading strategy, which is realized on GPU.

\section{CONCLUSION.}

China's energy production and consumption pattern shows a typical reverse distribution, which means that resources are mainly distributed in the west of China but consumed mainly in the east. Even hydropower, wind power and other clean energy shows the same pattern. For example, hydropower and wind power resources are mainly distributed in the southwest and northwest of China (including solar resources in Xinjiang). This pattern provide favourable conditions for large-scale electricity production, but also caused the difficulties of electricity transmission and consumption.Other users should not be affected when Microgrid connect into large grids, which requires accurate information of Microgrid sources, system performance, predictable load characteristics of both running 
connected and off-grid. But now, this work is not good enough.we research and develop the practical mathematic modeling adopted by multi-functional smart agent system. To migrate the smart agent system into Cloud based platform for function integration.Futhermore,we will research and develop Random Matrix Theory based on big data and machine learning and establish solid data analysis foundation for smart agent system.

\section{REFERENCES}

[1] Rigole T., Vanthournout K., De Brabandere K., Deconinck G.: "Agents Controlling the Electric Power Infrastructure," Int. Journal of Critical Infrastructures IJCIS (Inderscience), Vol.4, No. 1/2, 2008, 2008; pp. 96-109.

[2] D Singh, CK Reddy.A survey on platforms for big data analytics. Springer Journal of Big Data,( 2014), pp.1-20

[3] M News.Storage System for 'Big Data' Speeds Access to Information Communications of the Acm, (2014)

[4] C Fang. Using NVMe Gen3 PCIe SSD Cards in High-density Servers for High-performance Big Data Transfer Over Multiple Network Channels, Slac Document Server, (2015) 